\title{
PANORAMA DE LA INVESTIGACIÓN SOBRE RELACIÓN CON EL SABER EN ARGENTINA
}

\section{PANORAMA OF RESEARCH ON THE RELATIONSHIP WITH KNOWLEDGE IN ARGENTINA}

\author{
Autores: \\ ${ }^{1}$ Soledad Vercellino \\ Docente investigadora del Centro Interdisciplinario de Estudios sobre Derechos, Inclusión y Sociedad \\ (CIEDIS) Universidad Nacional de Río Negro (UNRN) y Centro Universitario Regional Zona Atlántica \\ (CURZA) - Universidad Nacional del Comahue.
}

Viedma, Río Negro, Argentina

\section{Contato do autor principal}

El Caín 234, (8500), Viedma, Río Negro. Email: svercelllino@unrn.edu.ar 


\title{
PANORAMA DE LA INVESTIGACIÓN SOBRE RELACIÓN CON EL SABER EN ARGENTINA
}

\author{
PANORAMA OF RESEARCH ON THE RELATIONSHIP WITH KNOWLEDGE IN ARGENTINA
}

\author{
${ }^{1}$ Soledad Vercellino
}

\begin{abstract}
RESUMO
A noção de "relação com o saber" tem sido assumida como central em nossa pesquisa, capaz de organizar questionamentos e buscas em torno do problema da aprendizagem. Nesta comunicação, cuja primeira versão foi exposta na tabela desenvolvida no âmbito do XII Colóquio Internacional "Educação e Contemporaneidade" (Aracaju, setembro de 2018), basearemos porque consideramos uma categoria fértil investigar o aprendizado escolar e suas vicissitudes e explicaremos o desenvolvimento incipiente, mas vigoroso, da noção no campo da pesquisa educacional na Argentina.
\end{abstract}

Palavras-chave: RELAÇÃO COM CONHECIMENTO; APRENDIZAGEM; PESQUISA EDUCACIONAL.

\begin{abstract}
The notion of relationship with knowledge has been assumed as central to our research, capable of organizing questions and searches around the problem of learning. In this communication, the first version of which was exposed in the table 'The relationship with knowledge: research landscape in Argentina, Brazil and Uruguay', developed within the framework of the XII International Colloquium 'Education and Contemporaneity' (Aracajú, Brazil, September 2018 ), we will base why we consider it a fertile category to investigate school learning and its vicissitudes and we will account for the incipient, but vigorous, development of the notion in the field of educational research in Argentina.
\end{abstract}

Key words: RELATION WITH KNOWLEDGE; LEARNING; EDUCATIONAL RESEARCH.

\section{RESUMEN}

La noción de "relación con el saber" ha sido asumida como central en nuestra investigación, capaz de organizar cuestionamientos y búsquedas en torno al problema del aprendizaje. En esta comunicación, cuya primera versión fue expuesta en la tabla desarrollada en el ámbito del XII Coloquio Internacional "Educación y Contemporaneidad" (Aracaju, septiembre de 2018), basamos porque consideramos una categoría fértil investigar el aprendizaje escolar y sus vicisitudes y explicaremos el desarrollo incipiente, pero vigoroso, de la noción en el campo de la investigación educativa en Argentina.

Palabras clave: RELACIÓN CON CONOCIMIENTO; APRENDIZAJE; INVESTIGACIÓN EDUCACIONAL.

\section{INTRODUCCIÓN}

La noción de relación con el saber la venimos asumiendo como central en nuestras investigaciones, susceptible de organizar los interrogantes y búsquedas en torno a la problemática del aprender.

Una primer versión de esta comunicación fue expuesta en la mesa 'A relação com o saber: panorama das pesquisas na Argentina, Brasil e Uruguai' desarrollada en el marco del XII Colóquio Internacional "Educação e Contemporaneidade" (Aracajú, Brasil, septiembre del 2018). Esta mesa, propuesta y coordinada por el prof. Dilson Cavalcanti, con el apoyo del Prof. Bernard Charlot, coordinador general del Coloquio, se realizó en el marco de una serie de colaboraciones e intercambios que investigadores de los tres países latinoamericanos venimos realizando. 
En este artículo fundamentaremos por qué consideramos a noción de relación con el saber una categoría fértil para investigar el aprendizaje escolar y sus vicisitudes y daremos cuenta del incipiente, pero vigoroso, desarrollo de la misma en el ámbito de la investigación educativa en Argentina.

\section{La relación con el saber: una categoría para investigar el aprendizaje escolar.}

El aprendizaje escolar o también llamado aprendizaje formal ha sido objeto de investigación de diferentes disciplinas y desde posiciones epistemológicas y metodológicas diversas. Las ciencias de la educación, la psicología, la psicopedagogía, la sociología, la antropología, la filosofía, la epistemología, la economía, las neurociencias, el campo de la inteligencia artificial, entre otros, se interrogan, investigan y producen conocimiento en torno al aprendizaje y, específicamente, el que acontece en el escenario escolar. Así, la actividad intelectual y emocional involucrada en el aprender, la significatividad socio-cultural y personal de los aprendizajes, los procesos de enseñanza, la mediación subjetiva del docente y de las tecnologías escolares, la cultura y la dinámica de las institución escolar y su función más o menos reproductora y de transmisión ideológica o más o menos emancipadora, el papel de otros agentes socializadores; el análisis epistemológico de la naturaleza, estructura y organización del conocimiento científico y de su traducción en conocimiento escolar y personal; la productividad y eficacia de los factores de aula, escuela y contexto sobre el rendimiento educativo de los alumnos, la base neurobiológica de los procesos de aprendizaje y un largo etcétera, son algunas de las formas en que las ciencias sociales y humanas han abordado la cuestión del aprender y sus vicisitudes.

La 'caja negra' (GOOD Y BROPHY, 1990) del aprendizaje, es decir, los denominados -según la corriente o teoría de la cual se trate- como procesos mentales, cognoscitivos, inteligentes, emocionales o afectivos implicados en la apropiación y recreación de los saberes disponibles y dispuestos por lo escolar, ha dado lugar a más de medio siglo de investigación en el campo de la psicología educacional y disciplinas afines.

Tales investigaciones advierten sobre la existencia de diferentes procesos cognoscitivos que se ponen en juego al aprender1 (BENGOCHEA GARÍN, 2003; COLL Y OTROS 2001). Algunas posturas focalizan en el individuo, exclusivamente en la interacción del sujeto con el objeto de conocimiento y en los procesos de recreación y producción de conocimiento que

\footnotetext{
${ }^{1}$ Junto a los aprendizajes de naturaleza exclusivamente constructivista, es decir, aquellos que siguen procesos de reestructuración o reorganización de los conocimientos previos y que comportan cambios conceptuales, se identifican aprendizajes de tipo conceptual que permiten interpretar, clasificar y categorizar hechos dentro de un marco conceptual y aprendizajes de información verbal en los que se incorporan datos a la memoria, por ello, son de carácter memorístico, asociativo, mecánico y repetitivo
} 
tienen lugar en el sujeto (perspectiva presente en el conductismo, en algunas aplicaciones piagetianas y en el enfoque del procesamiento de la información). Otras posiciones focalizan en el contexto social donde se produce la situación de aprendizaje (posición iniciada por Vygotsky y continuada, entre otros, por ROGOFF, 1993, BENGOCHEA GARÍN, 2003). Estos trabajos sostienen la tesis de que los procesos psicológicos superiores implicados en el aprender, tienen un origen histórico y social, se dan en situación, en contexto, incluso, aquellos más avanzados, se adquieren en el seno de procesos instituidos de "socialización" específicos como son los procesos de escolarización. También las teorías del aprendizaje por "reestructuración" o constructivistas han puesto el foco en la ocurrencia de ciertos procesos de aprendizaje en el contexto del aula focalizando en los de mediación, en el sentido y pertinencia social de los aprendizajes, etc.

En otros estudios se analiza las características cooperativas o competitivas de los grupos de pares; la influencia de la tarea o contenido, la intervención del adulto educador (ROGOFF, 1993). El carácter social del aprendizaje escolar también ha sido puesto de relevancia en los estudios - psicológicos, didácticos y epistemológicos- que advierten la coexistencia de conocimientos que surgen de procesos, procedimientos y escenarios diferentes, a saber: el conocimiento escolar, el conocimiento científico y el conocimiento cotidiano. El foco en estas investigaciones se ubica en las relaciones que esos conocimientos establecen entre sí en el ámbito escolar: ora de sustitución, ora de yuxtaposición, ora de convivencia más o menos conflictiva (CHEVALLARD, 2000).

También reparan en los procesos de metacognición o autoconciencia sobre los propios aprendizajes: sobre el sí mismo como aprendiente, sobre las actividades o tareas que se realizan, sobre los recursos con los que se cuenta, etc. Esta dimensión reguladora de la metacognición es, según BENGOCHEA GARÍN (2003) objeto de gran parte de la investigación educativa europea.

Finalmente, los estudios refieren a la intervención en el aprendizaje escolar de los genéricamente denominados 'factores personales': se insiste en su relevancia e influencia en los procesos y productos del aprendizaje, así como también en su numerosidad. Aquí se incluyen estilos de aprendizaje y de pensamiento, expectativas y representaciones de sí mismo y de otros, auto concepto y autoestima académicos, etc. (BENGOCHEA GARÍN, 2003).

Ahora bien, en nuestras investigaciones afrontamos el desafío de volver sobre ese complejo campo de problemas que es el aprendizaje escolar, entendido como un trabajo psíquico, singular y situado. Y lo hacemos ayudadas por los aportes heurísticos de las teorías sobre la relación con el saber. Esta noción, que desde hace más de 30 años es utilizada o 
invocada en el campo de las investigaciones en educación, permite organizar algunos de nuestros interrogantes y búsquedas y nos permite afrontar el desafío de volver sobre ese complejo campo de problemas que es el aprendizaje escolar, entendido como un trabajo psíquico, singular y situado

Entendemos que la noción de 'relación con el saber' cuenta con la capacidad heurística de articular en el análisis del aprendizaje, las relaciones y múltiples influencias e imbricaciones de las dimensiones destacadas por las teorías del aprendizaje escolar, es decir, como el aprender acontece en la encrucijada de diferentes procesos cognitivos y metacognitivos con su anclaje sociohistórico y situacional; el vínculo del sujeto aprendiente con los objetos de aprendizaje; las características epistemológicas de los objetos de aprendizaje; la singularidad del psiquismo de quien aprende.

Se trata de una noción de carácter multidisciplinar, pues los estudios sobre la 'relación con el saber' se vienen desarrollando desde el campo del psicoanálisis, la sociología y la didáctica. Asimismo es una noción que ha permitido abordar diversos objetos de investigación: el aprendizaje formal en espacios escolares y, pero también el aprendizaje que acontece en ámbitos no escolares, como el laboral; los procesos de recreación de saberes focalizando en el alumno o en el adulto educador y en diferentes niveles educativos, desde maternal hasta la universidad y los procesos - más bien epistemológicos y didácticos- de transformación de los saberes.

Adicionalmente, los estudios de la relación con el saber, han permitido desarrollar diferentes técnicas de investigación, como por el ejemplo, el denominado 'método clínico' en los estudios psicoanalíticos o los 'balances de saber' en la propuesta sociológica; técnicas que se vinculan a que esos estudios enfatizan en diferentes dimensiones de análisis: procesos libidinales vinculados al aprender, tipo de actividades intelectuales comprometidas en ese proceso; sentidos adjudicados al saber; formas institucionales de organización y presentación de los saberes, por citar algunas.

No obstante esta diversidad, en todos los casos, con esta noción se intenta "unificar, comprender o explicar lo que atañe a los aprendizajes, los conocimientos y las múltiples dificultades con que se tropieza" (BEILLEROT, BLANCHARD LAVILLE y MOSCONI 1998:44). Los investigadores coinciden en asumir que el aprendizaje escolar, sus vicisitudes y atolladeros, quedan cifrados en la encrucijada entre cierta disposición psíquica constituida en la historia singular de cada sujeto y cierta oferta institucional de saberes, configurada sociohistóricamente y significada singularmente. 


\section{El estado de la investigación sobre la relación con el saber en Argentina.}

A partir de la década del 90 se desarrolla una fuerte investigación empírica en el ámbito internacional que indaga la relación con el saber de los alumnos en los diferentes ámbitos del sistema educativo. El rastreo bibliográfico realizado ha permitido advertir que la categoría relación con el saber es desarrollada y cuenta con amplia difusión en Francia y otros países de habla francesa, al punto de registrarse varios eventos académicos y científicos dedicados a su análisis, como es el caso del Symposium "Relación con el saber: del concepto a sus usos", desarrollado en el marco del Congrès International d'Actualité de la Recherche en Education et en Formation, realizado en Strasbourg en el año 2007 y del Dossier Rapport au savoir de la Revista Espirit Critique en su edición del año 2013, codirigido por investigadores de la Universidad Católica del Oeste de Angers, Francia y de la Universidad de Québec Trois Rivières (Canadá).

Entre las investigaciones francófonas dedicadas al desarrollo de la noción, caben citarse a KALALI (2007); MAIRONE (2007); POULIOT ET AL. (2010); VENTURINI, CALMETTES, AMADE-ESCOT y TERRISE (2007); HATCHUEL (2009); GAGNON, (2011); ALLAIN (2011); POGGI Y MUSARD, (2014); C. BEAUCHER, V. BEAUCHER y MOREAU (2013); THERRIAULT, BADER y NDONG (2013); MOREAU (2013), RICHARD-BOSSEZ (2013) y la tunesina MILED (2012). La noción aparece con muy poca frecuencia en la bibliografía en idioma inglés (POULIOT et al., 2010). En idioma español se han identificado investigaciones del país ibérico (HERNÁNDEZ Y TORT, 2009), de Colombia (ZAMBRANO, 2013), Chile (QUILAQUEO y QUINTRIQUEO, 2010; QUINTRIQUEO y TORRES, 2012) y Uruguay (GRIECO, 2013).

En el caso de Brasil, una reciente tesis doctoral (BESERRA CAVALCANTI, 2015), mapea el uso de la noción de relación con el saber en la literatura científica de ese país, estableciendo que la misma es "uma das noções-chave para o estudo e pesquisa de problemáticas ligadas [...] ao campo da Educação e do Ensino, especialmente, de Ciências e Matemática [una de las nociones claves para el estudio e investigación de las problemáticas ligadas al campo de la Educación y de la Enseñanza, especialmente, de las Ciencias y la Matemática] (BESERRA CAVALCANTI, 2015, p.10). El citado estudio permitió relevar un conjunto de 241 tesis, disertaciones, artículos y actas de congresos en cuyo titulado se referencia a la noción. Algunos de los trabajos desarrollados en el ámbito brasilero son: CHARLOT (2013. 2010, 2002, 2000), SOUZA (2017, 2016, 2015), SOUZA Y CHARLOT (2016), FRANCO (2016); MENDES Y BACCON, (2016), DIEB (2015, 2008), TRÓPIA (2015), TRÓPIA Y CALDEIRA (2011), OLIVEIRA (2015), SILVA Y ALMEIDA (2015), LIMA (2015), LIMA, 
PASSOS, ARRUDA Y DOHL (2015), CONTI, PASSOS Y ARRUDA (2014), COSTA (2014), LUCAS, PASSOS Y ARRUDA (2013), ARRUDA, LIMA Y PASSOS (2011), BACCON Y ARRUDA (2010), KLEIN, ARRUDA, PASSOS Y ZAPPAROLI (2010), BACCON Y ARRUDA (2015), PASSOS, MARTINS, Y ARRUDA (2005), ARRUDA Y UENO (2005), BETTI, MAFFEI Y USHINOHAMA (2015), BETTI Y USHINOHAMA (2014), MENDES (2015), PARENTI Y REIS (2014), REIS (2013, 2012), POMPEU (2013), FREITAS (2012); NEVES (2012), BICALHO (2009), SILVA Y TEIXEIRA (2009 Y 2007), DA SILVA (2008), BITTAR (2005) Y REGO (2000) .

La radicación de Bernard Charlot en ese país desde hace unos años ha significado un estímulo para el desarrollo de la noción, coronado con la creación de la REPERES: la Red de Pesquisa sobre a sobre a Relação com o Saber (Red de investigación sobre la Relación con el Saber), espacio que se propone promover la investigación sobre la relación con el saber, permitir la comunicación entre los investigadores (formados o principiantes) y proporcionarles apoyo.

Con respecto a la Argentina, se han hallado más de una docena de trabajos, que podemos organizar de la siguiente manera:

Tabla $\mathbf{N}^{\circ}$ 1: Grupos de investigación que refieren a la relación con el saber en Argentina.

\begin{tabular}{|c|c|c|c|}
\hline Identificación $^{2}$ & Inscripción institucional & Principales trabajos & Foco de interés \\
\hline $\begin{array}{l}\text { Grupo Patagonia } \\
\text { Norte }\end{array}$ & 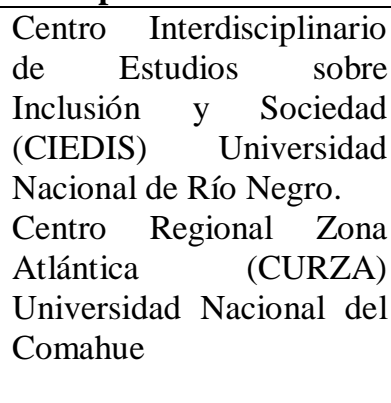 & $\begin{array}{l}\text { VERCELLINO et al, } 2018 . \\
\text { VERCELLINO, } 2015 \\
\text { VERCELLINO, VAN } \\
\text { DEN HEUVEL } \\
\text { GUERREIRO, } \\
\text { VERCELLINO, } 2014 \\
\text { ANDRADE, 2018; } \\
\text { TARRUELLA, 2018 } \\
\text { CARDINALE, } 2018\end{array}$ & $\begin{array}{l}\text { Fertilidad teórica de la } \\
\text { noción para la } \\
\text { investigación de los } \\
\text { aprendizajes escolares. } \\
\text { Relación con el saber de } \\
\text { estudiantes que finalizan la } \\
\text { educación primaria. } \\
\text { Relación con el saber de } \\
\text { estudiantes que ingresan a } \\
\text { la universidad. }\end{array}$ \\
\hline Grupo Córdoba I & $\begin{array}{ll}\text { Grupo del } & \text { Centro de } \\
\text { Estudios } & \text { Avanzados } \\
\text { Universidad } & \text { Nacional de } \\
\text { Córdoba } & \end{array}$ & $\begin{array}{l}\text { ARCANIO, } \\
\text { FALAVIGNA Y SOLER, } \\
2013 \\
\text { FALAVIGNA } \\
\text { ARCANIO, } 2011\end{array}$ & $\begin{array}{l}\text { Relación con el saber de } \\
\text { estudiantes que ingresan a } \\
\text { la universidad }\end{array}$ \\
\hline $\begin{array}{ll}\text { Grupo } & \text { de } \\
\text { Didáctica de } & \text { la } \\
\text { Matemática } & \end{array}$ & $\begin{array}{l}\text { Instituto } \\
\text { Investigaciones } \\
\text { Humanidades y Ciencias } \\
\text { Sociales- en } \\
\text { Nacional de La Plata }\end{array}$ & $\begin{array}{l}\text { BROITMAN, 2012a } \\
\text { 2012b; BROITMAN } \\
\text { CHARLOT, 2014. }\end{array}$ & $\begin{array}{l}\text { Relación con los saberes } \\
\text { matemáticos, en particular } \\
\text { en la educación de adultos } \\
\text { que inician su escolaridad } \\
\text { primaria }\end{array}$ \\
\hline $\begin{array}{l}\text { Grupo Córdoba- } \\
\text { Escuela } \\
\text { secundaria }\end{array}$ & $\begin{array}{l}\text { Facultad de Filosofía y } \\
\text { Humanidades. } \\
\text { Universidad Nacional de } \\
\text { Córdoba }\end{array}$ & UANINI et al, 2015 & $\begin{array}{l}\text { Relación con el saber y } \\
\text { escuela secundaria, con } \\
\text { énfasis en las políticas } \\
\text { educativas y curriculares } \\
\text { (Uanini et al, 2015). }\end{array}$ \\
\hline
\end{tabular}

\footnotetext{
${ }^{2}$ Estas formas de nominación se realizan a los fines de identificar grupos o equipos de trabajo, con diferentes niveles de institucionalización y continuidad en sus tareas, pero que en algún momento de su producción han abordado o están abordando investigaciones recurriendo a la potencia heurística de la noción.
} 


\begin{tabular}{|c|c|c|c|}
\hline Grupo CEM & $\begin{array}{l}\text { Centro de Estudios } \\
\text { Multidisciplinarios } \\
(\mathrm{CEM}) .\end{array}$ & $\begin{array}{l}\text { DIKER, 2007; FRIGERIO } \\
\text { Y DIKER, } 2005\end{array}$ & $\begin{array}{l}\text { Condiciones de enseñanza } \\
\text { que posibilitan otras } \\
\text { relaciones con el saber. } \\
\text { Análisis de contextos no } \\
\text { escolares }\end{array}$ \\
\hline $\begin{array}{l}\text { Estudios sobre la } \\
\text { relación con el } \\
\text { saber del docente }\end{array}$ & $\begin{array}{l}\text { Facultad de Filosofía de la } \\
\text { Universidad de Buenos } \\
\text { Aires. } \\
\text { Facultad de Filosofía y } \\
\text { Humanidades de la } \\
\text { Universidad Nacional de } \\
\text { Córdoba } \\
\text { Universidad Nacional del } \\
\text { Sur. }\end{array}$ & $\begin{array}{l}\text { TENAGLIA, } \\
\text { ARDILES Y BORIOLI, } \\
2010 ; \quad \text { JURE, 2010; } \\
\text { MONETTI, 2003 }\end{array}$ & $\begin{array}{l}\text { Relación con el saber } \\
\text { disciplinar y con el saber } \\
\text { didáctico }\end{array}$ \\
\hline
\end{tabular}

Fuente: propia.

A partir de la revisión de literatura realizada ${ }^{3}$ se advierte que algunas de esas indagaciones son de carácter teórico (en el sentido que GOHIER, 1998; DEMO, 2009 definen este tipo de indagación). Es el caso de los desarrollados por FALAVIGNA Y ARCANIO (2011) y VERCELLINO (2015). Realizan un trabajo más bien descriptivo y da cuenta del devenir de este concepto en las tres escuelas ya referenciadas: indican la red de conceptos propios de cada desarrollo, los principales representantes y grupos académicos que desarrollan investigaciones desde ese concepto (FALAVIGNA Y ARCANIO, 2011), dando cabal idea de que existen diferentes escuelas que desarrollan investigaciones en torno a dicha noción.

Las investigaciones empíricas, por su parte, se referencian en algunas de las tres escuelas ya nombradas o en más de una de ellas. Así TENAGLIA (2011) recurre a los desarrollos que desde la perspectiva psicoanalítica realizan los investigadores del Equipo de la Universidad de París X Nanterre, (BEILLEROT et al., 1998). Por su parte BROITMAN (2012a y 2012b) y BROITMAN Y CHARLOT (2014) harán lo propio con los desarrollos que desde la sociología crítica, realiza el Equipo Escol, en la Universidad París VIII- Sant Denis, en particular Bernard Charlot. Las investigaciones que focalizan en el adulto educador (TENAGLIA, 2011; MONETTI, 2003; JURE, 2010) se apoyan en los desarrollos del Equipo de Irem de AixMarseille, (CHEVALLARD, 2000).

A hora bien, más allá de los debates teórico-epistemológicos entre la perspectiva psicoanalítica y la sociológica, no son pocas las investigaciones que conforman sus marcos conceptuales con los aportes de ambas corrientes. Es el caso de los trabajos de MONETTI (2003); DIKER (2007); FRIGERIO Y DIKER (2005); FALAVIGNA Y ARCANIO (2011) y

\footnotetext{
${ }^{3}$ Una primera revisión bibliográfica que analiza los distintos desplazamientos teóricos que se han suscitado con la noción de 'relación con el saber' en su utilización efectiva en trabajos de investigación empírica y teórica desde distintos campos disciplinares puede consultarse en: VERCELLINO (2015). La misma ha sido revisada y ampliada para esta tesis.
} 
ARCANIO, FALAVIGNA Y SOLER (2013).

En relación al contexto educativo en el que desarrollan las investigaciones, si bien la mayor cantidad de investigaciones focalizan en la escuela primaria común (VERCELLINO et al, 2018); también se registran trabajos con alumnos del nivel medio (TENAGLIA, 2011, UANINI et al 2015;), del nivel universitario (MONETTI, 2003, FALAVIGNA Y ARCANIO, 2011 y ARCANIO, FALAVIGNA Y SOLER, 2013), adultos que realizan estudios primarios (BROITMAN, 2012a y 2012b y BROITMAN Y CHARLOT, 2014) e incluso, otras investigaciones (DIKER, 2007; FRIGERIO Y DIKER, 2005; GUERREIRO, 2016) abordará esta noción para analizar procesos educativos en instancias no escolares.

La revisión de esas investigaciones nos permite advertir que algunas de ellas focalizarán el estudio en los procesos, que podríamos denominar, primarios de construcción de la relación con el saber, aquellos que en términos genéricos, se desarrollan en el ámbito familiar. Más apoyados en categorías de la sociología (como la de origen socioprofesional), de la psicología educacional (como las de alianzas co-parentales, valores y estilos educativos familiares) o socioantropológicas (como las de socialización, prácticas socioculturales e interculturalidad) los trabajos reseñados dan cuenta de las marcas -más o menos posibilitadoras- que los procesos de subjetivación (hominización, según CHARLOT, 2008) dejan en la forma en que el sujeto se relaciona con el saber. Asimismo dan cuenta que el ingreso y tránsito por instituciones educativas, con sus propios reservorios de significaciones, lógicas de funcionamiento y exigencias hacia el sujeto, constituyen ocasiones para conmover, interpelar o reforzar las modalidades de relación del alumno con el saber.

Mientras otros (la mayoría) analizarán la relación con el saber del alumno en situaciones de aprendizajes que se desarrollan en instituciones, en su mayoría, escolares, es decir, indagarán la relación con el saber escolar.

\section{La investigación sobre la relación con el saber en la región Patagónica}

En la región de la Patagonia Norte, desde el 2013 venimos desarrollando estudios sobre la relación con el saber, enmarcados en tres proyectos de investigación. El primero, financiado por la Universidad del Comahue ha indagado la fertilidad teórica esta noción para la comprensión psicopedagógica de los aprendizajes escolares y sus problemas, identificando y problematizando los distintos desplazamientos teóricos que la misma ha tenido (PI V04/084 CURZA UNComa, 2013); luego, un segundo proyecto, en la misma universidad, denominado "La constitución de la relación con el saber en los alumnos del último ciclo del nivel primario y primero del nivel medio. Viedma, Río Negro” (PI V097 CURZA, UNComa, 2015), buscó profundizar el estudio de la noción y analizar los procesos secundarios de constitución de la 
relación con el saber en alumnos pertenecientes a escuelas urbana insertas en sectores de pobreza, identificando los sentidos que éstos otorgan a los saberes escolares y explorando cómo se configura la relación docente - alumno- saberes en el ámbito escolar.

Finalmente, a partir del 2017, exploramos la relación con el saber universitario de los alumnos que comienzan las carreras de ciencias aplicadas de la Universidad Nacional de Río Negro (UNRN). La investigación, que cuenta con el asesoramiento del Dr. Bernard Charlot procura más específicamente: caracterizar en términos socioeducativos a los alumnos ingresantes a esas carreras; identificar qué tipo de actividades intelectuales son demandadas a tales estudiantes al ingresar a las mismas y a cuáles éstos adjudican mayor significancia y reconstruir para ciertos casos los procesos singulares de relación con el saber (PI UNRN, 2017).

En Argentina, donde los textos del CREF fueron traducidos tempranamente y la obra de Charlot más recientemente, las investigaciones sobre la relación con el saber, inicialmente aisladas, se vuelven más numerosas y organizadas.

En mayo del 2018 hemos promovido la conformación de una mesa temática en el VII Congreso Nacional y V Internacional de Investigación Educativa 2018 de la Argentina, desarrollado en la ciudad de Cipolletti, Río Negro, titulada: "Los aportes de los estudios sobre la relación con el saber a la investigación educativa”. La misma, coordinada por investigadores argentinos y brasileros, contó con la presentación de 8 trabajos provenientes de distintos centros de investigación.

Asimismo, se ha publicado un libro titulado "La escuela y los (des)encuentros en la relación con el saber" (Editorial UNRN). En el mismo, tal como resalta el Prof. Charlot en su prólogo, procuramos abordar las cuestiones teóricas esenciales dando una muestra del valor heurístico de la noción de relación con el saber en la investigación de campo.

\section{El libro combina la reflexión teórica y la investigación de campo.}

En relación a nuestras indagaciones teóricas en torno a la noción de relación con el saber: hemos realizado una amplia revisión de las investigaciones que recurren a esa categoría, lo que permite visibilizar los desplazamientos teóricos de la misma y las posibilidades que brinda para el análisis psicopedagógico de los aprendizajes escolares. Asimismo, hemos propuesto revisitar los orígenes psicoanalíticos de la noción en la obra de Jacques Lacan, origen que entiendo ha sido olvidado por gran parte de la investigación en la temática. Esa propuesta es especialmente trabajada por Nora TARRUELLA (2018) quien en su trabajo vuelve a la lectura psicoanalítica para profundizar en la noción de saber y su vínculo con la verdad, sosteniendo como hipótesis que la creencia es una modalidad de relación con el saber cuya 
conformación es el saldo de la operación de renegación, de allí su consistencia y resistencia a ser modificada. Lidia CARDINALE (2018) por su parte, explora la controversia entre Bernard Charlot y Francoise Dubet, entendiendo que la misma permite "articular, refinar, modificar, integrar [sendos desarrollos y] constituyen una vía que conduce a revelar dimensiones ocultas y supuestos implícitos” (NUDLER, 2009, p.47) sobre la noción que nos ocupa.

Finalmente, Romina VAN DEN HEUVEL (2016, 2018), propone una indagación epistemológica, inspirada en la propuesta teórico-metodológica de Georges CANGUILHEM (1971, 1975, 2009), analizando los movimientos de conceptos producidos en dos contextos teóricos, disciplinares y sociales diferentes, a saber: el de la relación con el saber del psicoanalista francés BEILLEROT $(1996,1998)$ y el de modalidad de aprendizaje de la psicopedagoga argentina FERNÁNDEZ (1987).

\section{Las indagaciones empíricas que hemos realizado, son variadas.}

La investigación de Rocío ANDRADE (2018) analiza la relación con el saber que han construido adolescentes que cursan sus estudios en escuelas secundarias insertas en lof comunidades - mapuche, mapuche-tehuelche rurales y urbanas de la Provincia de Río Negro. La investigación, un estudio de casos cualitativo, concluye que los saberes y prácticas de saber comunitarios menos fragmentarios, más o menos ocultados, más o menos reivindicados, con escasez de ritos que sostengan su inscripción colectiva e individual (múltiples ceremonias ancestrales se han perdido, otras perduran o han mutado). Las ofertas del sistema educativo, si bien tienen en común haber sido diseñadas para impedir el desarraigo de sus comunidades, de su cultura, son cuestionadas por los adolescentes quienes denuncian el empobrecimiento de la oferta educativa. En ese contexto los jóvenes construyen una particular relación con el saber, que el texto describe minuciosamente.

Un equipo de psicopedagogas nos hemos abocado a los procesos secundarios de constitución de la relación del alumnado con el saber, focalizando en estudiantes que culminan el nivel primario y que inician el nivel medio en escuelas públicas de la provincia de Río Negro. Específicamente se identificaron y problematizaron los sentidos que el alumnado otorga a los saberes escolares, y más en general, a la escuela y a sus trayectos en ella: pasados y futuros. Analizamos tal relación pesquisando, en primer lugar, la relación epistémica con el saber, es decir, explorando con qué tipos de saberes (saberes objetos, actividades, modos de relación) el alumnado interactúa y que procesos de aprendizajes se dan con este (objetivación, apropiación, dominio, etc.). Al considerar que aprender tiene sentido en referencia "a la historia del sujeto, sus relaciones con los otros, a la imagen que tiene de sí mismo y a aquella que quiere dar a los 
otros" (CHARLOT, 2008, p. 117), atendimos la dimensión social e identitaria de la relación con el saber (VERCELLINO et al 2017, 2018).

Advertimos que estos alumnos y su contexto escolar jerarquizan como saber fundamental - y condición para la enseñanza de otros saberes- el dominio de las relaciones intersubjetivas en situación: es decir, saberes morales, políticos sobre como vincularse con los otros y consigo mismo en el ámbito escolar y no escolar. Ahora bien, la escuela promueve con estos saberes un modo de relación que se ajusta a lo que CHARLOT (2008) ha tipificado como “objetivación- denominación”. Esto significa que la relación con los saberes relacionales que dispone la escuela lejos de propiciar el dominio, el saber conducirse en situación, el saber actuar, instala al saber relacional en la figura de un saber -objeto, es decir, lo convierte en enunciados normativos sobre el buen conducirse y la relación con los demás. El saber relacional aparece entonces como "un existente en sí, en un universo de saberes distinto del mundo de la acción, de las percepciones, de las emociones" (CHARLOT, 2008, p. 112) que es dónde se aprende a relacionarse con los otros y consigo mismo.

También observamos que los alumnos dominan ciertas actividades propias de lo escolar (la escritura, la tecnología) pero lo hacen bajo cánones, criterios, normatividades no escolares. La escritura no responde a la lengua estándar, el uso de las TIC no es el previsto por el escolar.

En este punto, ante el desafío planteado por organismos gubernamentales y académicos respecto a la necesidad de que los alumnos logren apropiarse de los saberes que el sistema educativo se propone transmitir (POGGI, 2015), nuestra investigación se pregunta ¿la adquisición de qué tipos de saberes (objetos o conocimientos, dominios relacionales o de actividad) promueven las figuras del aprender que propone la escuela? ¿Resultan pertinentes dichas figuras para adquirir los saberes escolares que pretenden enseñar y acreditar? Creemos que estas preguntas pueden reubicarse en otros niveles del sistema educativo. Bajo esa conjetura estamos trabajando hoy en día en el nivel universitario.

Esas preguntas, asimismo, permiten correr el foco del déficit del alumno a la potencialidad de la intervención de la escuela; reconocer que la problemática se ubica no en la ausencia de oferta, sino en la necesidad de repensar la misma en término de las relaciones con el saber que esta promueve; ubicar la centralidad de las figuras del aprender que, más o menos reflexivamente, se promueven en la escuela y analizar que figuras del aprender se han instituido y cómo ellas mismas conspiran contra la posibilidad de aprender.

Estos alumnos solo identifican a la escuela y su familia como los lugares donde se aprende y en dónde se encuentran los adultos referentes del aprender. Los alumnos viven con 
intensidad su escolaridad (pasada, presente y futura), al punto que, por ejemplo el pasaje al nivel medio los conmueve e interpela. La imagen de sí que le devuelve su porvenir está signada por la dificultad, la angustia y una demanda de hospitalidad hacia el nuevo espacio escolar.

Pesquisar la relaciones con el saber que los alumnos reconocen como posibilitadoras, de gran valor e interés aun cuando se dan en ámbitos no escolares (como lo son para quienes participaron de esta investigación, el futbol, la música, otros deportes), da pistas de las condiciones necesarias para promover otras relaciones con el saber escolar: ser convocante en "mi mundo" con "otros"; ser un saber hacer de gran valor en la comunidad, ser una vía de reconocimiento, de identificación, ser un significante que hace lazo social.

Como nos advierte CHARLOT (2018), “más que una teoría ya construida, aunque elementos de teorización sean propuestos, "relación con el saber" define una nueva manera de preguntar, una nueva mirada sobre cosas cotidianas que, de cierta manera, "ya se sabe", pero que "uno ya no ve", tal vez un nuevo paradigma. Sin trabajo teórico, el trabajo de campo y la propia expresión de "relación con el saber" pueden perderse en descripciones que son finalmente banales. Sin los datos de campo, el discurso teórico corre el riesgo de atascarse en los debates académicos, sin que se perciba el poder explicativo que la mirada en términos de relación con el saber origina".

En esa tarea estamos.

\section{REFERENCIAS BIBLIOGRÁFICAS}

ANDRADE R. B. ¿Qué relación con el saber construyen adolescentes mapuches en secundarios insertos en comunidades mapuches? Tesis de pregrado. Universidad Nacional del Comahue. Viedma, Río Negro, 2016.

ARDILES, M. Y BORIOLI, G. Las huellas de la academia en los profesores de profesores. Algunos resultados del trabajo de campo. Praxis Educativa, 16(14), pp. 57-65, 2010.

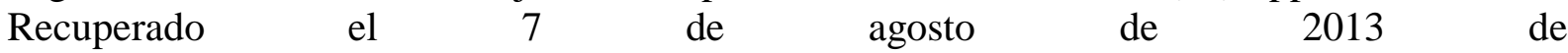
http://www.biblioteca.unlpam.edu.ar/pubpdf/praxis/n14a07ardiles.pdf

BEAUCHER, C., BEAUCHER, V. Y MOREAU, D. Contribution à l'opérationnalisation du concept de rapport au savoir. Revue Esprit Critique, 17, pp. 6-29, 2013.

\section{BEILLEROT, J., BLANCHARD LAVILLE, C. Y MOSCONI, N. Saber y relación con el} saber. Buenos Aires: Paidós Educador, 1998

BENGOECHEA GARIN, P. Aprendizaje escolar: una cuestión de permanente debate, una aproximación a su definición. Aula Abierta, 81, 2003.

BICALHO, M. G. P. Y SOUZA, M. C. R. F. Relação com o saber de estudantes universitários: Aprendizagens e processos. Educaçao e Pesquisa, Revista da Faculdade de Educação da 
Universidade de São Paulo, 40(3), pp. 617-635, 2014.

BROITMAN, C. Adultos que inician la escolaridad: Sus conocimientos aritméticos y la relación que establecen con el saber y con las matemáticas [en línea]. Tesis de doctorado. Universidad Nacional de La Plata. Facultad de Humanidades y Ciencias de la Educación. La Plata, 2012a. En Memoria Académica. Disponible en: http://www.memoria.fahce.unlp.edu.ar/tesis/te.899/te.899.pdf

BROITMAN, C. Relación con el saber y recursos matemáticos de adultos que inician la escolaridad primaria. Revista del Instituto de Investigaciones en Ciencias de la Educación, 2012b, no 32, p. 39-54.

BROITMAN, C.; CHARLOT, B. La relación con el saber. Un estudio con adultos que inician la escolaridad. Educación matemática, 2014, vol. 26, no 3, p. 7-35.

CAVALCANTI, J. D. B. A. Noção de relaçãoao saber: história e epistemologia, panorama do contexto francófono e mapeamento de suautilizaçãona literatura científica brasileira. Tesis de doctorado. Área de Concentração: Educação Matemática, Universidad Federal Rural de Pernambuco, Brasil, 2015.

CHARLOT, B. La question du rapportau savoir: convergences et différences entre deux aproches. Savoirs, (10), pp. 37-43, 2006. DOI: 10.3917/savo.010.0037.

CHARLOT, B. La relación con el saber, formación de maestros y profesores,educación y globalización. Montevideo: Editorial Trilce , 2008 ${ }^{\mathrm{a}}$

CHARLOT, B. La relación con el saber. Elementos para una teoría. Buenos Aires:Libros del Zorzal, 2008b.

CHEVALLARD, Y. La transposición didáctica. Del saber sabio al saber enseñado. Buenos Aires: Aique Grupo Editor, 2000.

COLL, César; MARTÍN, Elena; ONRUBIA, Javier. La evaluación del aprendizaje escolar: dimensiones psicológicas, pedagógicas y sociales. C. Coll, J. Palacios y A. Marchesi (Comps), Desarrollo psicológico y Educación, 2001, vol. 2, p. 549-574.

DA SILVA, V. A. Relação com o saber na aprendizagem matemática: uma contribuição para areflexão didática sobre as práticas educativas. Revista Brasileira de Educação, 13(37), p. $151,2008$.

DEMO, P. Elementos metodológicos da pesquisa participante. Repensando a pesquisa participante. São Paulo: Brasiliense, 1999.

DIKER, G. Y FRIGERIO, G. ¿Es posible promover otra relación con el saber? Reflexiones en torno del proyecto DAS. En: Las formas de lo escolar, 225-244. Buenos Aires: Del Estante Editorial, 2007.

FALAVIGNA, C. Y ARCANIO, M. Redes teóricas en torno a la relación con el saber. Elementos para el análisis de una noción en construcción. Revista IRICE (CONICETUNR), (22), pp. 7-16, 2011. 
FERNÁNDEZ, A. La inteligencia atrapada. Buenos Aires: Ediciones Nueva Visión, 1987

FRIGERIO, G. Y DIKER, G. Acerca del saber, de la relación de saber y de las condiciones para el aprender. Informe de investigación elaborado para UNESCO/OREALC, 2005. Recuperado el 7 de agosto de 2013 de http://unesdoc.unesco.org/images/0014/001492/149270s.pdf

GAGNON, M. Penser la question des rapport saux savoirs en éducation: clarification et besoin de recherches conceptuelles. Les ateliers de l'éthique The Ethics Forum. La Revue du CREUM, 6(1), pp. 30-42, 2011. Recuperado el 7 de agosto de 2013 de http://creum.umontreal.ca/wp-content/uploads/2011/06/pdf_04_Gagnon.pdf

GARNIER, A. Le rapport au savoir de l'enseignant, révélateur du fonctionnement du système didactique. Journal de la Recherche surl'Intervention en Éducation Physique et Sport. l'Institut Universitaire de formation des Maîtres de l'Université Franche12 Comté, 2008.

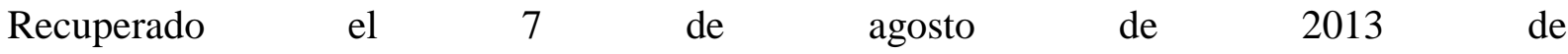
http://www.fcomte.iufm.fr/ejrieps/ejournal14/Garnier\%20eJ\%2014.pdf

GOHIER, C. La recherche theórique en sciences humaines: réflexiones sur la validité d'énoncés théoriques en education. Revue des sciences d l'éducation, 24(2), pp. 267- 284, 1998. Recuperado de http://id.erudit.org/iderudit/502011ar

GOOD, Thomas L.; BROPHY, Jere E. Educational psychology: A realistic approach. Longman/Addison Wesley Longman, 1990.

GRIECO, L. La dimensión grupal de la relación con el saber. Estudio de un caso en el escenario de la educación superior. Querencia, 14, pp. 72-99, 2013. Recuperado el 7 de agosto de 2013 de http://revista.psico.edu.uy/index.php/querencia/article/view/160/90

GUERREIRO, M. La relación de sujetos adultos con el saber corporal en el marco de la enseñanza y el aprendizaje del método pilates. Tesis de pregrado. Centro Universitario Regional Zona Atlántica, Universidad Nacional del Comahue, Viedma, 2016.

JURE, E. Los docentes noveles y su relación con el saber que enseñan. En II Congreso Internacional sobre profesorado principiante e inserción profesional a la docencia. Buenos Aires, $2010 . \quad$ Recuperado de http://cedoc.infd.edu.ar/noveles/principiantes/5/INV_5_Jure_LOS_DOCENTES_NO

KALALI, F. Rapport au savoir: bilan sur la place du sujet dans les different travaux. En Symposium: Rapportsau (x) savoir (s): du concept aux usages. Congrès international Actualité de la Recherche en Education et en Formation, Strasbourg. 2007. Recuperado el 7 de agosto de 2013 de http://www.congresintaref.org/actes_pdf/AREF2007_Faouzia_KALALI_422.pdf

MILED, A. B. Éducation familiale et rapport au savoir chez des garçons et des filles tunisiens de première année d'école primaire: une approche interactionniste sociale. Tesis de doctorado. Université Toulouse le Mirail, Toulouse II, 2012. Recuperado el 7 de agosto de 2013 de http://tel.archives-ouvertes.fr/tel-00727300/

MONETTI, E.La relación con el saber didáctico del docente de la Universidad Nacional del Sur. En Congreso Latinoamericano de Educación Superior en el Siglo, Vol. 21, pp. 18-19, 2003. 
NUDLER, O. Espacios controversiales. Hacia un modelo de cambio filosófico y científico. Buenos Aires: Miño y Dávila, 2009.

POGGI, M. P., Y MUSARD, M. Rapport au savoir en EPS à l'école primaire. Recherches en éducation. 2014.

POGGI, M., ALMIRÓN, G., AREA MOREIRA, M., BILAGHER, M., BRENER, G., FUMAGALLI, L., HEPP, P., RAVELA, P. Y TIANA FERRER, A. Mejorar los aprendizajes en la educación obligatoria: políticas y actores. Ciudad Autónoma de Buenos Aires: Instituto Internacional de Planeamiento de la Educación IIPEIIPE-Unesco, 2015.

QUILAQUEO, D. Y QUINTRIQUEO, S. Saberes educativos mapuches: un análisis desde la perspectiva de los kimches. Polis, 9(26), pp. 337-360, 2010.

QUINTRIQUEO MILLÁN, S. Y TORRES CUEVAS, H. Distancia entre el conocimiento mapuche y el conocimiento escolar en contexto mapuche. Revista electrónica de investigación educativa, 14(1), pp. 16-33, 2012. Recuperado el 3 de febrero de 2014 de http://redie.uabc.mx/vol14no1/contenido-quintriqueotorres.html.

RICHARD-BOSSEZ, A. Saisir le rapport aux savoirs en actes à l'école maternelle: éléments de réflexion conceptuels et empiriques. Rapport au savoir, 123, 2013.

ROGOFF, B. Children's guided participation and participatory appropriation in sociocultural activity. Development in context: Acting and thinking in specific environments, 1993, p. 121-153.

TENAGLIA, G. Los rasgos de la relación con el saber del docente en el espacio de formación profesional (taller) en la enseñanza técnica. Estudio clínico. Tesis de maestría. Universidad de Buenos Aires, 2011. Recuperado el 12 de agosto de 2013 de www.filo.uba.ar/contenidos/investigación/institutos/Iice/ANUARIO_2011/textos/30.T

THERRIAULT, G., BADER, B. Y NODNG ANGOE, C. L'apport de la notion de rapport(s) $\mathrm{au}(\mathrm{x})$ savoir(s) en education aux sciences et en formation initiale et continue des enseignants du secondaire: des exemples au Quebec et au Gabon. Revue Esprit critique, (17), pp. 70-94. Universidad de París X 2013. Centre de recherche en éducation et formation. Parisnanterre. Recuperado de: https://cref.parisnanterre.fr/

VAN DEN HEUVEL, R. Movimientos conceptuales en el campo psicopedagógico. Análisis de las relaciones entre conceptos propuestos por Jacky Beillerot y Alicia Fernández. Tesis de pregrado. Centro Universitario Regional Zona Atlántica, Universidad Nacional del Comahue, Viedma, 2016.

VAN DEN HEUVEL, R. Y VERCELLINO, S. La movilidad de conceptos en el campo psicopedagógico: una aproximación a las relaciones entre conceptos propuestos por Jacky Beillerot y Alicia Fernández. Revista Pilquen, 12(1), pp. 1-13.2015

VEntURINI, P., CAlmettes, B., AMADE-ESCOT, C. Y TERRISE, A.. Analyse didactique des practieus de 'enseignement de la physiqued'un professeur eexperimentée. Aster, 45, pp. 211-234, 2007. Recuperado el 7 de febrero de 2014 de 
https://www.google.com.ar/\#q=Venturini+\%2B+Calmettes

VERCELLINO, S (comp). La escuela y los (des)encuentros con el saber. Editorial UNRN, 2018.

VERCELLINO, S. La «relación con el saber»: revisitando los comienzos del concepto. Revista Pilquen, 16(11), pp. 1-8, 2014.

VERCELLINO, S. Revisión bibliográfica sobre la relación con el saber. Desplazamientos teóricos y posibilidades para el análisis psicopedagógico de los aprendizajes escolares. Revista Electrónica Educare, 19(2), pp. 53-82, 2015. Recuperado de http://www.revistas.una.ac.cr/index.php/EDUCARE/rt/printerFriendly/6579/6670

VERCELLINO, S., VAN DEN HEUVEL, R. Y GUERREIRO, M. Relação com o conhecimento: a revisão da literatura sobre e possibilidades teóricas para a análisepsicoeducacional da aprendizagem escolar. Revista Psicopedagogia, 31(96), pp. 275-288, 2014. Recuperado de http://pepsic.bvsalud.org/pdf/psicoped/v31n96/05.pdf

ZAMBRANO LEAL, A. Relación con el saber, fracaso/éxito escolar y estrategias de enseñanza-aprendizaje. Actualidades Pedagógicas, 61, pp. 27-43, 2013. Recuperado el 05 de agosto de $2013 \mathrm{de}$ http://revistas.lasalle.edu.co/index.php/ap/article/view/2329

Submetido em: 19.10.2018

Aceito em: 23.11.2018

Publicado em: 30.12.2018 